\begin{tabular}{|c|l|}
\hline Title & Measurement of electrically induced shear strain in a chiral smectic liquid-crystal elastomer \\
\hline Author(s) & Na, Y ang Ho; A buraya, Y uki; Orihara, Hiroshi; Hiraoka, Kazuy uki \\
\hline Citation & $\begin{array}{l}\text { Physical Review E, 83(6), 061709 } \\
\text { https://doi.org/_0.1103/PhysRevE.83.061709 }\end{array}$ \\
\hline Issue Date & 2011-06 \\
\hline Doc URL & http://hdl.handle.net/2115/46791 \\
\hline Rights & @2011American Physical Society \\
\hline Type & article \\
\hline File Information & PRE83_6_061709.pdf \\
\hline
\end{tabular}

Instructions for use 


\title{
Measurement of electrically induced shear strain in a chiral smectic liquid-crystal elastomer
}

\author{
Yang Ho Na, ${ }^{1}$ Yuki Aburaya, ${ }^{1}$ Hiroshi Orihara, ${ }^{1, *}$ and Kazuyuki Hiraoka ${ }^{2}$ \\ ${ }^{1}$ Division of Applied Physics, Graduate School of Engineering, Hokkaido University, North 13 West 8, Kita-ku, Sapporo 060-8628, Japan \\ ${ }^{2}$ Department of Life Science and Sustainable Chemistry, Tokyo Polytechnic University, 1583 Iiyama, Atsugi 243-0297, Japan
}

(Received 7 March 2011; revised manuscript received 13 May 2011; published 27 June 2011)

\begin{abstract}
The mechanical response to electrical stimulation was investigated in a chiral smectic elastomer. The twodimensional strain tensor in an elastomer film was precisely measured by tracking fluorescent beads dispersed on the film. Shear deformation in the film was clearly observed when an electric field was applied perpendicular to the film surface. The temperature dependence of the strain tensor was also investigated, and the origin of the electric-field-induced shear strain in the chiral smectic- $C$ phase was mainly attributed to the Nambu-Goldstone mode.
\end{abstract}

DOI: 10.1103/PhysRevE.83.061709

PACS number(s): 61.30.-v, 61.41.+e, 77.65.-j

\section{INTRODUCTION}

The possibility of a new class of liquid-crystal materials has been put forward by de Gennes, and liquid-crystal elastomers (LCEs) are unique materials with anisotropy of liquid-crystal phases and elasticity of polymer networks [1-3]. The distinguishing feature of LCEs is a reversible macroscopic shape change under external stimulation [4-15]. Among these materials, chiral smectic elastomers have attracted increasing attention because of properties such as ferroelectricity, piezoelectricity, and second-harmonic generation, which are ascribed to the symmetry breaking induced by the chiral liquidcrystalline moiety [11-20]. In particular, the piezoelectricity may be important in applications.

In LCEs, changes in the orientation of the director are coupled with deformation of the polymer network. In chiral smectic LCEs, furthermore, these changes are coupled with electric field, resulting in piezoelectricity. The orientational changes are classified into several modes in chiral smectic phases. Chiral smectic LCEs deform according to the modes that are excited by an electric field. Figure 1(a) shows the soft mode in the chiral smectic- $A\left(\operatorname{Sm} A^{*}\right)$ phase. When an electric field is applied, a tilt angle $\delta \theta$ is induced, followed by shrinkage of the smectic layer. This is known as the electroclinic effect [21]. Figures 1(b) and 1(c) respectively show the amplitude and phase (Nambu-Goldstone) modes in the chiral smectic- $C\left(\mathrm{SmC}^{*}\right)$ phase. When an electric field is applied to the $\mathrm{Sm} C^{*}$ phase, the mesogen (director) may change the tilt angle (amplitude mode), rotate around the layer normal (phase mode), or both, to orient the direction of the spontaneous polarization $P_{s}$ toward that of the electric field. If the amplitude mode in $\mathrm{Sm} C^{*}$ is excited by an electric field, shrinkage perpendicular to the smectic layers and shear deformation should be observed. On the other hand, for the phase mode, only shear deformation without any change in length along the layer normal should be observed. The phase mode is related to "soft deformation," by which LCEs can deform without energy cost through rotation of the director [16], [18]. In contrast, there are energy costs associated with changing the layer spacing and the tilt angle in the amplitude mode [Fig. 1(b)]. Therefore, soft deformation in the $\mathrm{Sm} C^{*}$

*orihara@eng.hokudai.ac.jp phase may be obtained by rotating the director on a cone around the layer normal, that is, by the phase mode [Fig. 1(c)]. The relationship between the modes and types of deformation will be discussed in detail later.

Some experimental studies have been reported on electrically induced deformation. Lehmann and colleagues reported that an ultrathin film of a ferroelectric LCE shrank by $4 \%$ in the thickness direction when subjected to a lateral electric field [13]. They proposed that the thickness change was caused by the electrically induced tilt of the director by the soft and amplitude modes in the $\operatorname{Sm} A^{*}$ and $\operatorname{Sm} C^{*}$ phases, respectively. Hiraoka et al. have also investigated electrically induced shrinkage and elongation along the layer normal in a monodomain $\mathrm{SmC}^{*}$ elastomer [15]. However, these previous studies showed no direct evidence for electrically induced shear deformation, which is expected to be directly related to the director change and thus should have been observed first. To determine which mode is responsible for the deformation, direct observation in the deformation plane should be carried out. In this article, we propose a new method that enables the displacement of each position in the deformation plane under an electric field to be directly measured and the strain tensor to be calculated. The soft deformation in a chiral smectic LCE is also examined.

\section{EXPERIMENT}

By following the procedure previously reported by Finkelmann [22], an elastomer was synthesized by the hydrosilylation reaction between liquid-crystalline side groups and a polysiloxane backbone. The elastomer film was prepared by spin casting using solution containing a polysiloxane, two mesogens, a cross-linker, and a Pt catalyst in toluene. See Ref. [15] for chemical structures and molar ratios of molecular components. The reaction was carried out under centrifugation, and the reaction vessel then was cooled to room temperature. The elastomer, swollen with toluene, was carefully removed from the vessel and was deformed uniaxially by stress loading in order to achieve uniform orientation of the director until the cross-linking reaction was completed. During the deformation process at room temperature, the toluene evaporated continuously from the sample, and the sample underwent phase transformations from the isotropic phase of 
(a)

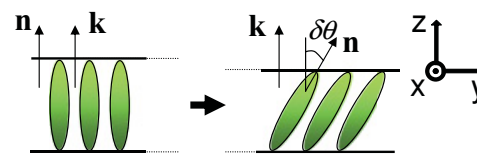

(b)

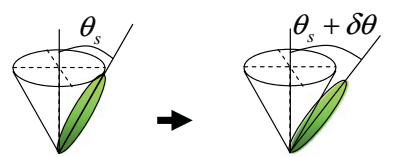

(c)

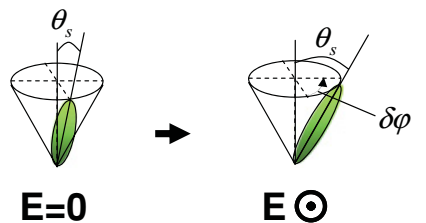

FIG. 1. (Color online) Expected behavior of mesogens by the contribution of (a) soft mode in $\mathrm{Sm} A^{*}$ phase, (b) amplitude mode, and (c) phase mode in $\mathrm{SmC}^{*}$ phase when an electric field is applied. $\mathbf{n}, \mathbf{k}$, and $\theta_{s}$ represent the director, layer normal, and smectic tilt angle, respectively. $\delta \theta$ and $\delta \varphi$ represent the field-induced changes in the tilt and the azimuthal angles, respectively.

the gel to the tilted smectic phase of the dry elastomer [23]. The elastomer shows the following phase sequence:

$$
g-6 \mathrm{Sm} X^{*} 32 \mathrm{Sm}^{*} 80 \mathrm{Sm}^{*} 115 \mathrm{I} \quad\left({ }^{\circ} \mathrm{C}\right) .
$$

The transition temperatures listed above were determined by differential scanning calorimetry and x-ray scattering, as already reported in previous papers [24,25]. The $\operatorname{Sm} X^{*}$ phase is a high-ordered tilted smectic phase. The results of $\mathrm{X}$-ray analysis suggested that this elastomer film in the $\mathrm{SmC}^{*}$ phase had a polydomain structure with the director of each domain being along the same direction, while in the $\operatorname{Sm} A^{*}$ phase it had the macroscopic monodomain structure [23]. Figure 2 shows a schematic of the molecular alignment in the sample obtained from $\mathrm{x}$-ray analysis. In the $\mathrm{SmC}^{*}$ phase, the directors are uniformly aligned along the direction of the deformation stress in sample preparation, but are tilted with respect to the layer normal. The layer normals are conically distributed around the director. Layer tilting might cause domain boundaries to form in the $\mathrm{SmC}^{*}$ phase of the elastomer, as shown in Fig. 2. In

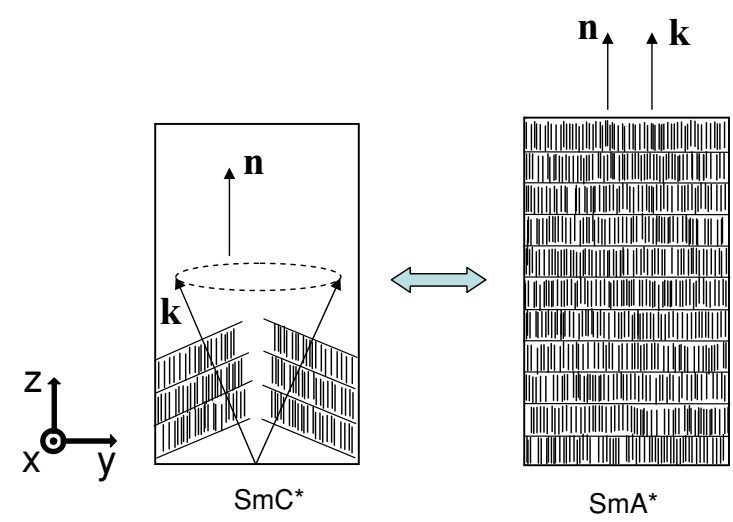

FIG. 2. (Color online) Schematic of molecular alignment in the sample in the $\mathrm{Sm} C^{*}$ and $\mathrm{Sm} A^{*}$ phases. $\mathbf{n}$ and $\mathbf{k}$ represent the director and layer normal, respectively.

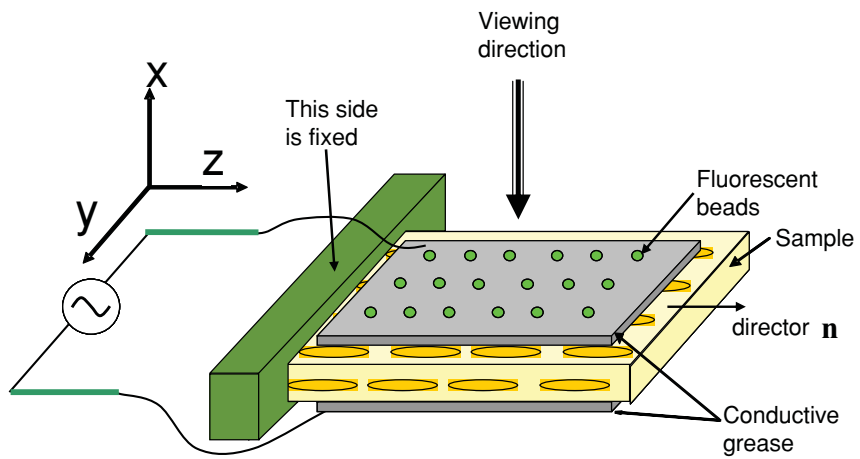

FIG. 3. (Color online) Schematic of experimental setup for electrically induced deformation studies. Right-hand side of the film was fixed. Left-hand side could move freely allowing small changes in the sample to be precisely monitored.

contrast, the layer tilting decreases with increasing temperature from the $\mathrm{Sm}^{*}$ phase to the $\mathrm{Sm} A^{*}$ phase, although the average direction of the director is almost independent of temperature, as shown on the right-hand side of Fig. 2.

Figure 3 shows the schematic of the experimental setup in the hot stage (HS1, Instec). The $z$ axis is taken along the direction of the director, and the $x$ axis is perpendicular to the LCE film. Electric field was applied parallel to the $x$ axis. The sample film was mounted on the hot stage for temperature regulation during measurements. Before each measurement, the sample was held for at least $20 \mathrm{~min}$ at the measurement temperature. The temperature was controlled within $0.1{ }^{\circ} \mathrm{C}$. Conductive grease (Circuit Works) was thinly coated on both surfaces of the elastomer sheet with a sample size of $6 \times 4 \times 0.4 \mathrm{~mm}^{3}$. The thickness of the grease layer was about $80 \mu \mathrm{m}$. Then, fluorescent beads (F8823, Molecular Probes) were homogeneously dispersed on the grease of the upper side. The movement of the fluorescent beads under an electric field was monitored with a CCD camera (QICAM, Qimaging) attached to a microscope (BX51, Olympus) to calculate the field-induced strain of elastomer film. In this experiment, therefore, the fluorescent beads should follow the deformation of the elastomer when subjected to the electric field. It can be thought that if the thickness of the grease layer is smaller than the linear dimension of the area covered by the grease, the elastomer deformation may be faithfully captured with the beads for a uniform strain. We have actually checked it by using an elastic tape with a thin grease layer partially coated on it. The tape was slightly elongated and the displacements of beads on the grease and the tape were compared to confirm the validity of our measurements.

Figure 4 shows a raw image taken from a typical experimental movie for our measurements. The size of image was $320 \times 240 \mu \mathrm{m}^{2}(1392 \times 1040$ pixels $)$. The whole intensity range was from 0 to 255 analog-to-digital units. As shown in Fig. 4, 10 fluorescent beads were selected, and the positions of the beads then were determined by pattern matching. The time dependence of the displacements and the strain tensor were calculated from the data on the $y$ and $z$ coordinates of 10 beads at different locations. The bead positions were obtained by an image analysis method. The accuracy of displacement is about $20 \mathrm{~nm}$ ( 0.1 pixels). From the $y$ and $z$ coordinates of 10 beads, 


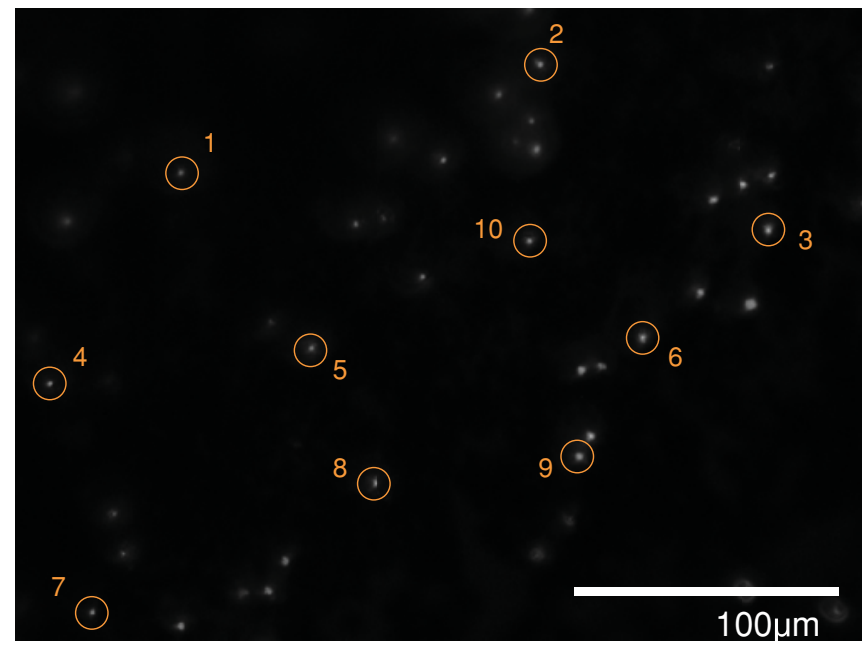

FIG. 4. (Color online) Image taken from a typical experimental movie $\left(45^{\circ} \mathrm{C}\right)$. Inserted numbers are given to identify the beads which were chosen for position measurement.

all the components of the two-dimensional strain tensor were calculated.

\section{RESULTS AND DISCUSSION}

The positions of 10 beads were traced when a sinusoidal electric field of $E_{0}=1.25 \mathrm{~V} / \mu \mathrm{m}$ in amplitude and $f=0.1 \mathrm{~Hz}$ was applied at $45{ }^{\circ} \mathrm{C}$ in the $\mathrm{SmC}^{*}$ phase. Figure 5(a) shows a typical time course of the displacement for one bead. Both the $z$ and $y$ components were clearly roughly proportional to the applied electric field, but the amplitudes were different: $0.2 \mu \mathrm{m}$ and $1.0 \mu \mathrm{m}$ for the $z$ and $y$ components, respectively. Other beads also showed the same sinusoidal behavior with the same phase, but with different amplitudes. The direction of deformation depends on the polarity of the applied electric field, indicating that spontaneous polarization is related to the deformation, not the dielectric anisotropy, as pointed out by Hiraoka et al. [15].

Next, the time dependence of the strain tensor was calculated from the displacements of several beads at different locations. Assuming spatially uniform deformation in the LCE, the two-dimensional strain tensor $\mathbf{D}(t)$ is given by

$$
\delta \mathbf{r}_{i}(t)=\delta \mathbf{r}_{i}(0)+\mathbf{D}(t) \delta \mathbf{r}_{i}(0)
$$

with

$$
\delta \mathbf{r}_{i}(t)=\mathbf{r}_{i}(t)-\mathbf{r}_{G}(t),
$$

where $\mathbf{r}_{i}(t)$ is the position of the $i$ th bead at time $t, \mathbf{r}_{G}(t)$ is the center of mass for the measured beads, and $\delta \mathbf{r}_{i}(t)$ is the relative position of the $i$ th bead from the center of mass. All the components of $\mathbf{D}(t)$ were determined by the least-squares method. Then, a pure strain tensor $\mathbf{S}(t)$ is defined by using $\mathbf{D}(t)$ :

$$
\mathbf{S}(t)=\frac{1}{2}\left[\mathbf{D}(t)+\mathbf{D}(t)^{T}\right],
$$

where superscript $T$ indicates transpose. Figure 5(b) shows the components $S_{\alpha \beta}$ obtained from 10 bead positions. First, let us check the validity of our analysis in terms of the relative position of the $i$ th bead from the center of mass, $\delta \mathbf{r}_{i}(t)$.
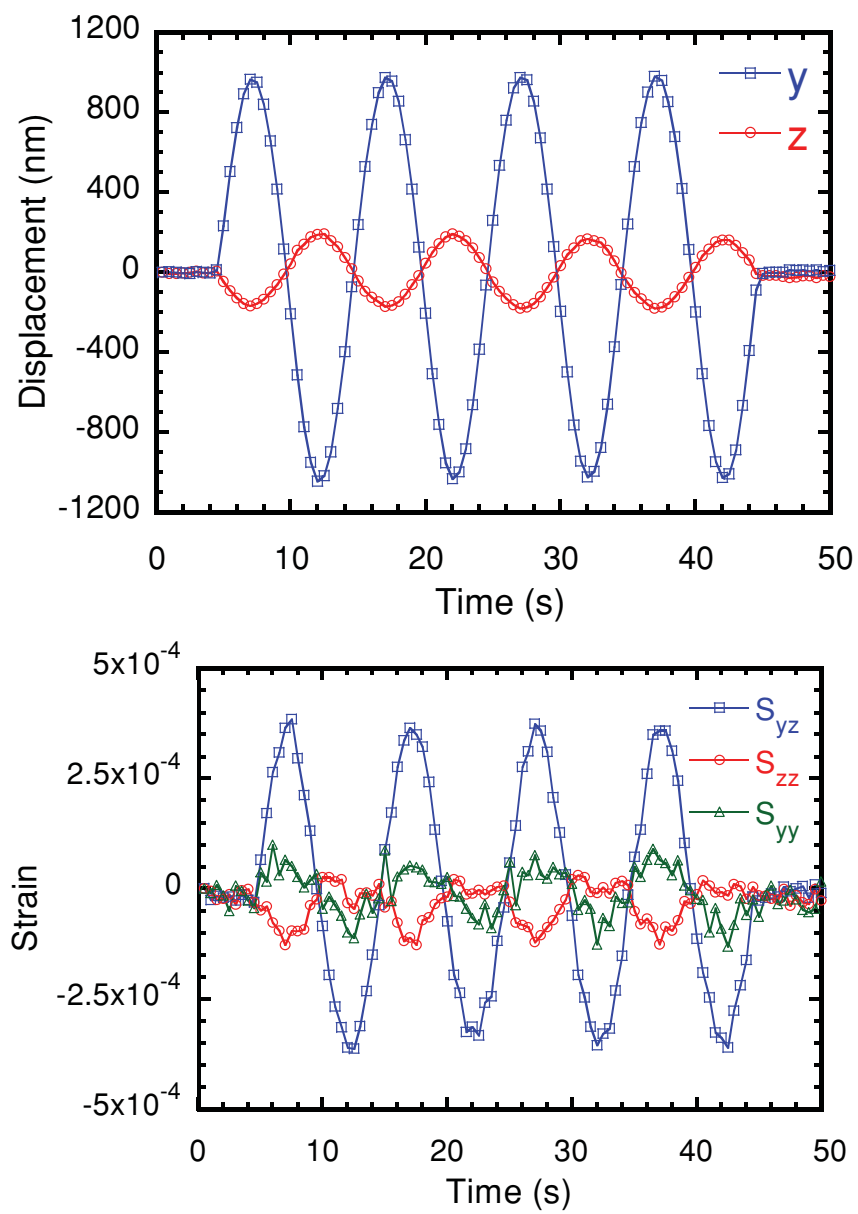

FIG. 5. (Color online) Time dependence of (a) displacement and (b) strain tensors at $45{ }^{\circ} \mathrm{C}$. Sinusoidal electric field $E=$ $E_{0} \sin [2 \pi f(t-5)]$ was applied with $f=0.1 \mathrm{~Hz}$ and $E_{0}=$ $1.25 \mathrm{~V} / \mu \mathrm{m}$ during 4 cycles. $E$ is zero at $t=0-5$ and $45-50 \mathrm{~s}$.

As shown in Fig. 6, we compared $\delta \mathbf{r}_{i}(t)$ obtained from the experiment with the one calculated from the obtained $\mathbf{D}(t)$. $\delta \mathbf{r}_{i}(t)$ obtained from the experiment is in good agreement with the value calculated from the obtained $\mathbf{D}(t)$, indicating that the assumption of uniform deformation is appropriate. In Fig. 5(b), the amplitude of $S_{y z}$ is much larger than that of the others, meaning that the shear strain is mainly induced by the electric field.

The dependence of the amplitude of each strain tensor component on temperature is shown in Fig. 7. The amplitudes of the first harmonics were obtained from the Fourier transform operation. $S_{y z}$ decreases with increasing temperature but the other components are small and nearly constant. As the temperature increases, the tilt angle of the director decreases and then the spontaneous polarization also decreases in the $\mathrm{SmC}^{*}$ phase. The decrease in $S_{y z}$ appears to be related to the decrease in the tilt angle, that is, the spontaneous polarization.

Next, let us consider which mode is responsible for the shear strain $S_{y z}$. The order parameters inducing the $\operatorname{Sm} A^{*}-\mathrm{Sm} C^{*}$ phase transition are $n_{x} n_{z}$ and $n_{y} n_{z}$ [26]. From symmetry considerations, we have approximate relations between the director and the strains, $S_{x z}=c n_{x} n_{z}$ and $S_{y z}=c n_{y} n_{z}$, with a constant $c$ for a small tilt angle $\theta$. The other components 

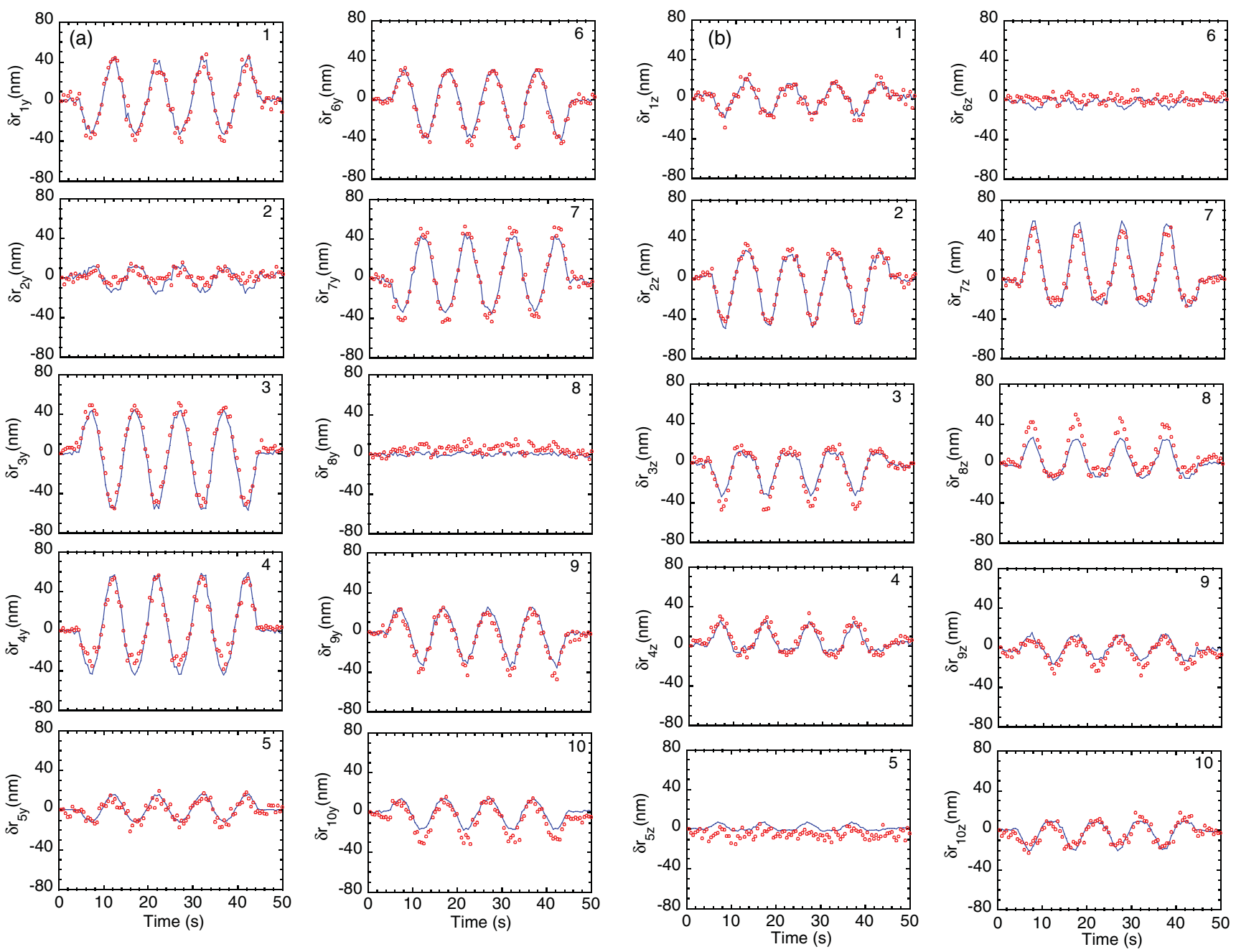

FIG. 6. (Color online) (a) $y$ and (b) $z$ components of the relative position of the $i$ th bead from the center of mass at $45^{\circ} \mathrm{C}$. The data obtained from the experiment (circles) and those calculated from the obtained data (line) are shown. Inserted numbers correspond to those shown in Fig. 4.

of the strain tensor are of second order with respect to $\theta$. It is readily seen that the constant $c$ should be positive. The strains are approximated as $S_{x z}=c \theta \cos \phi$ and $S_{y z}=c \theta \sin \phi$, where $\theta$ is the azimuthal angle from the $x$ axis. On the

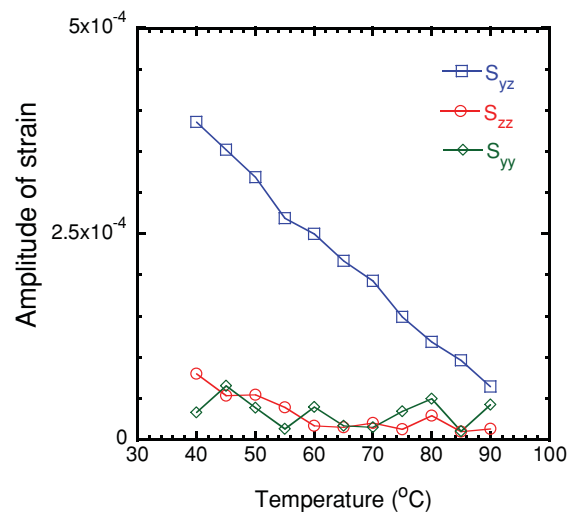

FIG. 7. (Color online) Dependence of amplitude of strain tensors on temperature. Applied electric field is the same as in Fig. 5. other hand, the tilt-induced polarization is expressed as $P_{x}=$ $-\mu \theta \sin \phi$ and $P_{y}=\mu \theta \cos \phi$, where $\mu$ is a constant, the sign of which depends on the sense of chirality [26]. From the electric interaction energy density $g_{\mathrm{el}}=-\mathbf{P} \cdot \mathbf{E}$, the forces conjugate to $\theta$ and $\phi$ become $f_{\theta}=\partial g_{\mathrm{el}} / \partial \theta=-\mu E \sin \phi$ and $f_{\phi}=\partial g_{\mathrm{el}} / \partial \phi=-\mu E \theta \cos \phi$, respectively, when we assume that the electric field is applied along the $x$ direction. The field-induced parts $\delta \theta$ and $\delta \phi$ can be written as $\delta \theta=\chi_{\theta} f_{\theta}$ and $\delta \phi=\chi_{\phi} f_{\phi}$, respectively, by using the susceptibility for the soft (amplitude) mode, $\chi_{\theta}$, and that for the phase mode, $\chi_{\phi}$. In the $\mathrm{SmC}^{*}$ phase $\chi_{\phi}$ may be very large and should become infinite in an ideal system because there is no restoring force for the phase mode. In our LCE, however, the fieldinduced deformation disappears after removing the electric field, indicating that there is no such phase mode with zero excitation energy. This is supported by the experimental result that the amplitude of $S_{y z}$ of our sample is the order of $10^{-4}$ as shown in Fig. 7, which is much smaller than $10^{-1}$ for a half rotation of the $c$ director with a spontaneous tilt angle of $10^{\circ}$. In contrast, $\chi_{\theta}$ is generally finite and increases as the transition point is approached [26]. It is conjectured that the effects of 
the relatively higher cross-linking density [27] and of defects between the polydomains [28] restrained the rotation relative to the polymer network in our LCE.

Assuming $\theta=\theta_{0}$ and $\phi=\phi_{0}$ at an equilibrium state without electric field in $\mathrm{SmC}^{*}$, the field-induced strains are given by

$$
\begin{aligned}
\delta S_{x z} & =c \cos \phi_{0} \delta \theta-c \theta_{0} \sin \phi_{0} \delta \phi \\
& =-\frac{1}{2} c \mu \sin 2 \phi_{0}\left(\chi_{\theta}-\chi_{\phi} \theta_{0}^{2}\right) E
\end{aligned}
$$

and

$$
\begin{aligned}
\delta S_{y z} & =c \sin \phi_{0} \delta \theta+c \theta_{0} \cos \phi_{0} \delta \phi \\
& =c \mu\left(\chi_{\theta} \sin ^{2} \phi_{0}+\chi_{\phi} \theta_{0}^{2} \cos ^{2} \phi_{0}\right) E,
\end{aligned}
$$

where we have used $\delta \theta=-\mu \chi_{\theta} E \sin \phi_{0}$ and $\delta \phi=$ $-\mu \chi_{\phi} E \theta_{0} \cos \phi_{0}$. From Eq. (4b) it is readily shown that the sign of $\delta S_{y z}$ is independent of $\phi_{0}$, indicating that each domain of a polydomain sample contributes to $\delta S_{y z}$ in an additive manner. On the contrary, $\delta S_{x z}$ may vanish by averaging over all the domains. It should be noted that we have assumed that the layer normal does not change for each domain in $\mathrm{SmC}^{*}$, while in our sample the director does not change, as described above. However, it is easily shown that Eqs. (4a) and (4b) are also applicable to the latter case insofar as the tilt angle is small. From Eq. (4b) it is shown that the contribution of the amplitude mode to $\delta S_{y z}$ increases as the transition point is approached, while the contribution of the phase mode may decrease because the tilt angle decreases and $\chi_{\phi}$ is thought not to have strong temperature dependence. The latter coincides with the experimental results. Thus, we can conclude that the phase mode is mainly responsible for the shear strain $\delta S_{y z}$ in the $\mathrm{SmC}^{*}$ phase, although it has finite energy cost and thus a restoring force. In the present experiment, we investigated the inverse piezoelectric effect. On the other hand, the direct piezoelectric effect was investigated by Finkelmann and coworkers [20,29]. They measured the piezoelectric coefficient $d_{x x x}$ in our coordinate system shown in Fig. 3, whereas in our experiment, $d_{x y z}$ is given by Eq. (4b), which is essential in the $\mathrm{Sm}^{*}$ phase

\section{SUMMARY}

We have described the macroscopic mechanical response of a freestanding ferroelectric liquid-crystal film to electric fields. Electrically induced shearing was clearly observed in chiral smectic phases. This shear deformation depended on the polarity of the applied electric field. It was also concluded that the contribution of the phase mode, that is, the coupling of the azimuthal rotation to the applied electric field, primarily caused the electrically induced deformation whose magnitude increased with decreasing temperature.

\section{ACKNOWLEDGMENTS}

K.H. thanks Professor Heino Finkelmann for his encouragement. The authors thank Professor Norihiko Nishiguchi for valuable discussions. This work was partially supported by Grants-in-Aid for Scientific Research [Priority Area "Soft Matter Physics" (Contract No. 463) and Wakate B (Contract No. 21740306)] from the Ministry of Education, Culture, Sports, Science, and Technology of Japan.
[1] P. G. de Gennes, Phys. Lett. A 28, 725 (1969).

[2] P. G. de Gennes, C. R. Acad. Sci. ser. B 281, 101 (1975).

[3] M. Warner and E. M. Terentjev, Liquid Crystal Elastomers, 2nd ed. (Clarendon, Oxford, 2007).

[4] E. Nishikawa, H. Finkelmann, and H. R. Brand, Macromol. Rapid Commun. 18, 65 (1997).

[5] D. L. Thomsen, P. Keller, J. Naciri, R. Pink, H. Jeon, D. Shenoy, and B. R. Ratna, Macromolecules 34, 5868 (2001).

[6] J. Harden et al., Appl. Phys. Lett. 96, 102907 (2010).

[7] R. Verduzco et al., J. Mater. Chem. 20, 8488 (2010).

[8] H. Finkelmann, E. Nishikawa, G. G. Pereira, and M. Warner, Phys. Rev. Lett. 87, 015501 (2001).

[9] Y. Yu, M. Nakano, and T. Ikeda, Nature (London) 425, 145 (2003).

[10] M. Camacho-Lopez, H. Finkelmann, P. Palffy-Muhoray, and M. Shelley, Nature Mater. 3, 307 (2004).

[11] D. Kramer and H. Finkelmann, Phys. Rev. E 78, 021704 (2008).

[12] R. Kohler, R. Stannarius, C. Tolksdorf, and R. Zentel, Appl. Phys. A 80, 381 (2005).

[13] W. Lehmann et al., Nature (London) 410, 447 (2001).

[14] C. M. Spillmann, B. R. Ratna, and J. Naciri, Appl. Phys. Lett. 90, 021911 (2007).

[15] K. Hiraoka, M. Kobayasi, R. Kazama, and H. Finkelmann, Macromolecules 42, 5600 (2009).
[16] O. Stenull and T. C. Lubensky, Phys. Rev. Lett. 94, 018304 (2005).

[17] J. M. Adams and M. Warner, Phys. Rev. E 73, 031706 (2006).

[18] J. M. Adams and M. Warner, Phys. Rev. E 79, 061704 (2009).

[19] P. Heinze and H. Finkelmann, Macromolecules 43, 6655 (2010).

[20] P. Papadopoulos, P. Heinze, H. Finkelmann, and F. Kremer, Macromolecules 43, 6666 (2010).

[21] S. Garoff and R. B. Meyer, Phys. Rev. Lett. 38, 848 (1977).

[22] H. Finkelmann, H. J. Kock, and G. Rehage, Makromol. Chem. Rapid Commun. 2, 317 (1981).

[23] K. Hiraoka, N. Tagawa, and K. Baba, Macromol. Chem. Phys. 209, 298 (2008).

[24] K. Hiraoka and H. Finkelmann, Macromol. Rapid Commun. 22, 456 (2001).

[25] K. Hiraoka, W. Sagano, T. Nose, and H. Finkelmann, Macromolecules 38, 7352 (2005).

[26] I. Musevic, R. Blinc, and B. Zeks, The Physics of Ferroelectric and Antiferroelectric Liquid Crystals (Singapore, World Scientific, 2000).

[27] W. Ren, P. J. McMullan, and A. C. Griffin, Phys. Status Solidi B 246, 2124 (2009).

[28] K. Urayama, E. Kohmon, M. Kojima, and T. Takigawa, Macromolecules 42, 4084 (2009).

[29] K. Hiraoka, P. Stein, and H. Finkelmann, Macromol. Chem. Phys. 205, 205 (2004). 